BY recording neuromagnetic events during aversive classical conditioning, we examined the extinction of a previously described conditioned response. Averaging over non-reinforced exposures to the conditioned stimulus revealed magnetic activity in the secondary somatosensory and insular cortices, appearing between 110 and $140 \mathrm{~ms}$ after the omitted unconditioned electric shock. We suggest this activity to be elicited by the discrepancy between shock expectancy and perceptual processes associated with the omission of the unconditioned stimulus, reflecting one of several brain processes in extinction.

Key words: Classical conditioning; Extinction; Magnetoencephalography; Somatosensory cortex

\section{Magnetic brain imaging of extinction processes in human classical conditioning}

\section{Gustav Wik, ${ }^{1,2}$ Thomas Elbert, $^{3}$ Mats Fredrikson, ${ }^{4}$ Manfried Hoke ${ }^{1}$ and Bernhard Ross ${ }^{1}$}

1'Institute for Experimental Audiology, University of Münster, Kardinal-von-Galen Ring 10, D-48129 Münster, Germany; ${ }^{2}$ Department of Clinical Neuroscience, Karolinska Institute and Hospital, Stockholm, Sweden; ${ }^{3}$ Department of Psychology, University of Konstanz, Konstanz, Germany; ${ }^{4}$ Department of Psychology, Uppsala University, Uppsala, Sweden

${ }^{\mathrm{CA}, 1}$ Corresponding Author and Address

\section{Introduction}

Classical conditioning results from a neutral stimulus repeatedly being paired with a stimulus that produces unconditioned responses (UCR). Learning is accomplished when the neutral stimulus itself elicits given reactions, termed conditioned responses (CR). Recently, we studied the acquisition phase of classical conditioning with magnetoencephalography (MEG), and reported that a CR produced by a visual conditioned stimulus (CS) was found in the primary somatosensory cortex; the receptive field of the electric shock unconditioned stimulus (UCS). ${ }^{1}$ In the present study, we report on MEG based measures from the extinction phase of classical conditioning, i.e. when the CS no longer is followed by UCS reinforcement.

Neural mechanisms mediating conditioned responses have been studied in humans with positron emission tomography (PET). ${ }^{2-4}$ A study of the specific eyeblink response, ${ }^{2}$ using an auditory warning signal as CS for an UCS corneal air puff, revealed redistribution of regional cerebral blood flow (rCBF) in the cerebellum, but also in the auditory cortex and cortices with associative function. A study of non-specific aversive conditioning with an auditory CS and electric shock UCS showed activa- tion predominantly of the right frontal and temporal cortices. ${ }^{3}$ Using a visual CS with an electric shock UCS, we applied PET to describe increased $\mathrm{rCBF}$ in subcortical and cortical brain areas. ${ }^{4}$ As well as bilateral activation in the thalamus, hypothalamus and the central gray of the midbrain, that study also revealed an asymmetrical increase of neural activity corresponding to the cortical SI representation of the electric shock UCS. In our previous MEG report, ${ }^{1}$ we demonstrated neuromagnetic activation in the SI appearing before UCS presentation, and suggested that this activation reflected part of a specific CR that served to reduce the noxious impact of the UCS. For the present study, we examined MEG recordings obtained during the extinction phase of that experiment, ${ }^{1}$ intending to elucidate CNS mechanisms associated with disappearance of the CR.

\section{Subjects and Methods}

A standard differential aversive conditioning paradigm was used in four right-handed healthy volunteers. ${ }^{1}$ Acquisition was accomplished as follows: when the reinforced visual conditioned stimulus (CS+) disappeared, after having been viewed by the subject for $2000 \mathrm{~ms}$, an intracutaneous electric shock UCS to the right middle finger was presented. A non- 
reinforced conditioned stimulus (CS-) served as the control cue. A picture of a spider and a sheep was for each subject randomly chosen to be CS+ or CS-, and shown with an intertrial interval varying between 3 and $10 \mathrm{~s}$ (mean $5 \mathrm{~s}$ ). Two runs containing $120 \mathrm{CS}+$ and 120 CS- trials each were performed. Results from this part of the experiment have been reported previously. ${ }^{1}$ To study extinction, a third run was then presented to the subjects. In the first two subjects this run was identical to the two acquisition runs, except that no UCS was given. Because no obvious neuromagnetic effects of shock expectancy or extinction could be determined from the average of the 120 CS exposure in this paradigm, we introduced three blocks of reinforcement, i.e. CS-UCS pairing, in the two remaining subjects; number III and IV in the previous study. ${ }^{1}$ Recordings from these reinforced blocks were not included in the data analysis. Each block consisted of five paired CS and UCS presentations, identical to those of the acquisition phase. One block appeared at the beginning of the extinction run, the second after $1 / 3$, and the third after $2 / 3$ of the run. Recordings were carried out in a magnetically shielded room with the subject fixated in a lateral position. ${ }^{1}$ When the exact position of the sensor array and the individual head shape had been determined, magnetic fields over the left hemisphere were recorded using a 37-channel (Magnes ${ }^{\circledR}$ BTi) neuromagnetometer. ${ }^{5}$ Recordings with possible artifacts were discarded. MEG-data collected during the trials where separately averaged for the CS+, UCS, and control (CS-, non-UCS) epochs. ${ }^{1}$ A lowpass $20 \mathrm{~Hz}$ filter was applied, and a single equivalent current dipole (ECD) model was used to fit field distributions measured during the last $100 \mathrm{~ms}$ of CS, the omitted UCS, and corresponding control periods. ${ }^{1}$ Data were sampled during at total of $1280 \mathrm{~s}$. To further elucidate extinction processes over time, we divided the extinction block, and made a separate analysis of its first and second $600 \mathrm{~s}$. The location and moment of the ECD were then estimated for each point in time. Confidence volumes were $<0.1 \mathrm{~cm}^{3}$. Dipole locations were described according to a head-based coordinate system with its medial-lateral axis ( $y$-axis) through the two meatuses and the origin in the midpoint between them. The anterior-posterior axis ( $x$-axis) was set from the origin to the nasion.

\section{Results}

In subjects III and IV, where reinforcement blocks had been inserted, dipolar neuromagnetic activity appeared after the omitted electric shock UCS. Figure 1 shows the response from a MEG sensor located over the supratemporal cortex in subject IV that

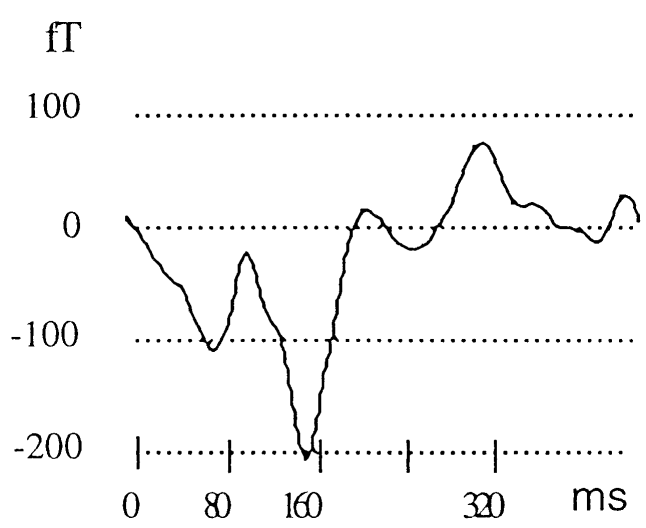

FIG. 1. Recording from a MEG sensor located over the supratemporal cortex during the extinction phase of classical conditioning. During acquisition, the electric shock unconditioned stimulus had been presented at $0 \mathrm{~ms}$.

has a first maximum about $80 \mathrm{~ms}$ and a second about $160 \mathrm{~ms}$ after the omitted electric shock UCS.

During the upstroke of the second component of the response, the superimposing to MRI images showed ECD model fits to be located in the secondary somatosensory (SII) and insular cortices. The SII location of dipoles is illustrated in Fig. 2.

Table 1 gives characteristics of neuromagnetic dipoles at $134 \mathrm{~ms}$ after the omitted UCS. Separate analysis of data obtained during the first $10 \mathrm{~min}$ of the extinction period resulted in similar dipole locations as for the whole period. For subject IV, the separate analysis revealed a better Goodness of Fit (0.990) and correlation (0.994), with corresponding values being lower $(0.925$ and 0.952$)$ for subject III (cf. Table 1) compared with the whole extinction

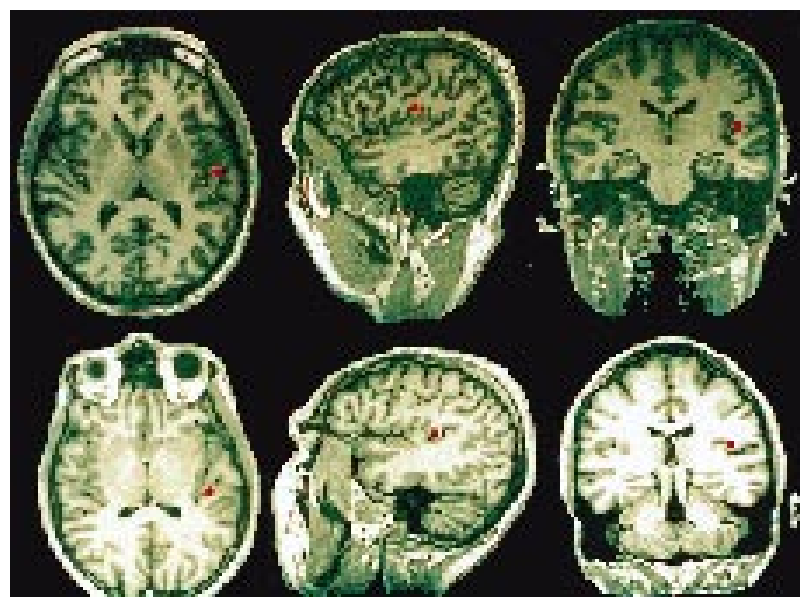

FIG. 2. The SII magnetic dipole location (marked as a dot) in subject III (above) and IV (below). In Subject III coordinates were: $x=1.6$; $y=4.3 ; z=6.1$ at $114 \mathrm{~ms}$ (displayed), then the dipole moved anteromedially into the insular cortex (cf. coordinates in Table 1). In subject IV the dipole location at $134 \mathrm{~ms}$ after UCS omission is displayed (cf. Table 1). 
Table 1. Characteristics and locations of neuromagnetic dipoles corresponding to the omitted electric shock unconditioned stimulus (omitted UCS) during the extinction phase of classical conditioning, with the unconditioned response (UCR) dipole as Ref. 1

\begin{tabular}{|c|c|c|c|c|}
\hline \multirow[t]{3}{*}{ Characteristics } & \multicolumn{4}{|c|}{ Subject ${ }^{a}$} \\
\hline & \multicolumn{2}{|c|}{ III } & \multicolumn{2}{|c|}{ IV } \\
\hline & $\begin{array}{l}\text { omitted } \\
\text { UCS }\end{array}$ & UCR & $\begin{array}{l}\text { omitted } \\
\text { UCS }\end{array}$ & UCR \\
\hline $\begin{array}{l}\text { ms after } \\
\text { stimulus onset }\end{array}$ & 134 & 77 & 134 & 77 \\
\hline Correlation $^{\mathrm{b}}$ & 0.985 & 0.995 & 0.984 & 0.996 \\
\hline $\begin{array}{l}\text { Goodness of fit } \\
\text { of the model }\end{array}$ & 0.990 & 0.994 & 0.964 & 0.994 \\
\hline $\begin{array}{l}\text { Root mean square } \\
\text { across channels }\end{array}$ & $\begin{array}{ll}\text { e } & \\
\text { s } & 61\end{array}$ & 123 & 97 & 263 \\
\hline $\begin{array}{l}\text { Anterior-posterior } \\
\text { location }(\mathrm{cm})\end{array}$ & $\begin{array}{r}r \\
2.63\end{array}$ & 2.08 & 0.79 & 2.49 \\
\hline $\begin{array}{l}\text { Medial-lateral } \\
\text { location }(\mathrm{cm})\end{array}$ & 3.02 & 2.42 & 4.25 & 3.37 \\
\hline $\begin{array}{l}\text { Superior-inferior } \\
\text { Iocation }(\mathrm{cm})\end{array}$ & 6.50 & 7.80 & 6.03 & 7.48 \\
\hline
\end{tabular}

asubject I and II are not included in the table because no obvious neuromagnetic effects of shock expectancy or extinction were observed. For subject III and IV, characteristics of the UCR have previously been reported. ${ }^{1}$

${ }^{b}$ The correlation between the measured and modeled field.

period. During the last $10 \mathrm{~min}$ of the extinction period only weak neuromagnetic activity, not suitable for further analysis, was seen.

\section{Discussion}

During the extinction phase of classical conditioning magnetic activity appeared between 110 and $140 \mathrm{~ms}$ after UCS omission. As determined by MRI overlays, dipoles were located in the upper bank of the Sylvian fissure, corresponding to the SII and the insular cortex. Accoring to Elbert et al., ${ }^{6}$ the SII dipole resulting from median nerve stimulation can be optimally described at about $120 \mathrm{~ms}$. In the present study, however, we gave no somatosensory stimulation during magnetic imaging, and the observed dipoles may instead reflect a reaction to UCS omission.

Although it is conceivable that the SII activation may be initialized from active SI signaling, this cannot be confirmed in the present data. At the time of possible SI activation, ${ }^{6}$ we found initial neuromagnetic activation that peaked about $80 \mathrm{~ms}$ after the omitted UCS presentation (cf. Fig. 1). Applying the ECD model to this component, however, did not result in reliable fits, leaving the origin of this early activation obscured. It is therefore possible that the SII and insular activation is a primary, rather than a secondary, reflection of UCS omission, and that it is elicited by the discrepancy between shock expectancy and processes associated with the perception of the omitted UCS.

During the CS period corresponding to the CR described in the previous report ${ }^{1}$ no localized activity was found. This indicates relatively fast extinction of the anticipatory component of classical fear conditioning, whereas components associated with UCS omission may be more persistent. Moreover, the averaging over 120 previously reinforced trials in the first two subjects revealed no obvious neuromagnetic effects of shock expectancy or extinction. To reestablish the conditioned association during the course of the run, reinforcement blocks were therefore added in the following two subjects. With reinforcement trials being omitted, the observed activation was produced from averaging over three blocks of 40 extinction trials each. Although this a non-standard approach to the study of extinction, it is not likely that the reinforcement blocks qualitatively altered extinction processes. Instead, the fact that no neuromagnetic activity was observed in the first two subjects probably reflects a fast response decay over the trials, indicating a high efficacy of processes associated with $\mathrm{CR}$ extinction. Further supporting this suggestion is the separate analysis of data sampled during the first and second part of extinction. Whereas data from the first $10 \mathrm{~min}$ produced characteristic dipoles in both subjects, no dipolar pattern whatsoever was seen during the last $10 \mathrm{~min}$.

Acknowledging the limited data base, we suggest that the observed neuromagnetic activation of the SII and the insula may reflect a first reaction to UCS omission, and that this reaction constitutes one of several processes responsible for CR extinction. The the role of the insular cortex has been emphasized in recent studies of pain. ${ }^{7}$ Besides receiving signaling from primary and secondary somatosensory areas, the insula is extensively interconnected with limbic, frontal, temporal, and parietal tertiary processing areas. ${ }^{89}$ Being a polymodal information convergence area for high-order control of pain and autonomic responses, ${ }^{10}$ we suggest an integrative role for the insular cortex in the extinction of an aversive conditioned response produced by noxious somatosensory stimulation. ${ }^{1}$

\section{Conclusion}

We studied the extinction phase of classical conditioning with MEG, and found neuromagnetic activity localized in the secondary somatosensory and insular cortices, between 110 and $140 \mathrm{~ms}$ after the omitted electric shock UCS. This activity may reflect the 
brain's reaction to the discrepancy between an expected but not perceived stimulus, and be one of several neural processes responsible for the extinction of conditioned responses.

\section{References}

1. Wik G, Elbert T, Fredrikson M et al. NeuroReport 7, 737-740 (1996).

2. Molchan SE, Sunderland T, Mclntosh AR et al. Proc Natl Acad Sci USA 91 8122-8126 (1994)

3. Hugdahl K, Berardi A, Thompson WL et al. NeuroReport 6, 1723-1728 (1995).

4. Fredrikson M, Wik G, Fisher H et al. NeuroReport, 7, 97-101 (1995).

5. Pantev C, Gallen C, Hampson S et al. Am J EEG Technol 31, 83-101 (1991).
6. Elbert T, Junghöfer M, Scholz B et al. Brain Topogr 7, 275-282 (1995).

7. Hsieh JC, Belfrage M, Stone-Elander S et al. Pain 63, 225-236 (1995).

8. Friedman DP, Murray EA, O'Neill JB et al. J Comp Neurol 252, 323-347 (1986).

9. Mesulam MM and Mufson EF. The insula of Reil in man and monkey. Architectonics, connectivity and function. In: Peters A and Jones EG, eds. Cerebral Cortex 4. New York: Plenum Press, 1985: 179-226.

10. Benarroch EE. Mayo Clin Proc 68, 988-1001 (1993).

ACKNOWLEDGEMENT: This work was supported by the Swedish Medical Research Council (10594), the Swedish Council for Research in Humanities and Social Sciences and the Deutsche Forschungsgemeinschaft. 